\title{
Les récepteurs-NMDA du glutamate permettent le modelage du système nerveux
}

Au cours du développement du système nerveux, la formation des circuits de connexions est soumise à un mécanisme d'erreurs/corrections et certaines synapses sont stabilisées alors que d'autres sont éliminées $\left(\mathrm{m} / \mathrm{s} n^{\circ} 4\right.$, vol. 6, p. 388). Il y a une vingtaine d'années, Changeux et Danchin avaient suggéré [1] que la stabilisation des synapses dépendait d'une interaction fonctionnelle entre les neurones pré- et postsynaptiques dans laquelle la mise en route d'une activité nerveuse possédant des caractéristiques précises jouait un rôle essentiel. Les mécanismes de cette interaction commencent à être dévoilés et tous les éléments convergent pour faire du récepteur-NMDA du glutamate un acteur majeur du modelage des systèmes de connexions au cours du développement du système nerveux.

Les premiers travaux, qui datent de la fin des années 1980, avaient démontré que le blocage des récepteurs-NMDA, par perfusion locale d'APV (acide D,L-2-amino-5phosphonovalérique) qui en est un antagoniste spécifique, perturbait distinctement la formation des connexions centrales des voies visuelles dans le tectum et dans le cortex cérébral (pour revue, voir [2]). La théorie, élaborée à partir de ces résultats et des caractéristiques connues des récepteurs du glutamate $\left(m / s n^{\circ} 10\right.$, vol. $\left.5, p .781\right)$, est que les neurones qui portent les récepteurs du glutamate de type NMDA et AMPA sont dépolarisés par la liaison du glutamate sur ces derniers. Il faut qu'un seuil de dépolarisation soit atteint pour que les canaux associés aux premiers soient rendus fonctionnels (par retrait des ions $\mathrm{Mg}^{2+}$ qui bloquent le canal), ce qui nécessiterait une convergence d'influx synchrones sur un même neurone. Le passage d'ions $\mathrm{Ca}^{2+}$ par le canal NMDA déclencherait alors une cascade de seconds messagers permettant, d'une part, des modifications métaboliques importantes dans la cellule postsynaptique, d'autre part, l'émergence d'un message agissant de façon rétrograde sur les neurones présynaptiques dont l'activité s'est révélée efficace (et pas sur les autres qui, à terme, seraient éliminés).

Deux travaux récents, réalisés dans d'autres régions du système nerveux, permettent de généraliser cette hypothèse et de mieux définir le rôle que le récepteur-NMDA pourrait jouer. Une équipe collaborative, formée autour de Tonegawa (MIT, MA, USA), a utilisé la technique de knock-out (invalidation génique par recombinaison homologue [3]) pour étudier le rôle du récepteur dans la différenciation cytoarchitecturale des noyaux trigéminaux du tronc cérébral [4]. La suppression par recombinaison homologue du gène codant pour NMDAR1, une sous-unité majeure du récepteur NMDA, conduit à la naissance d'animaux non viables, dont la mort intervient dans les 24 heures suivant l'accouchement. Il est toutefois possible de prolonger de 24 à 48 heures la survie de ces animaux, et cela a été suffisant pour observer l'absence de structuration des noyaux trigéminaux chez les souris privées de récepteur fonctionnel. Normalement, en effet, les neurones des noyaux trigéminaux forment des groupes fonctionnels distincts que l'on peut révéler simplement en marquant - par histochimie - l'activité cytochrome oxydase. La formation de ces groupes (appelés des barrelettes, par référence aux barrels mis en évidence dans le néocortex cérébral $-\mathrm{m} / \mathrm{s}$ $n^{\circ} 4$, vol. 6, p. 388), dépend de l'arrivée des projections trigémina- les et de la mise en route de l'activité nerveuse. Chez les souris NMDAR $1 \%$, cette mise en place ne semble pas avoir lieu. L'absence de récepteur-NMDA est la seule déficience observée (les afférences axonales sont présentes, les connexions sont établies et, pour le reste, fonctionnelles), ce qui est évidemment un argument très fort en faveur d'un rôle spécifique.

Quel est le rôle précis de ce récepteur et, plus précisément encore, est-ce celui prévu par l'hypothèse de Changeux et Danchin? Mariani (CNRS, Paris, France) a apporté récemment avec son équipe un élément essentiel dans cette direction [5]. Le modèle sur lequel travaille cette équipe, depuis des années, est celui de la rectification de la polyinnervation des cellules de Purkinje du cervelet par les fibres grimpantes dont l'origine se situe dans l'olive inférieure (figure 1). Dans une première phase développementale, chaque cellule de Purkinje reçoit en moyenne cinq fibres. Entre la naissance et le quinzième jour chez le rat, et en l'absence de mort neuronale dans l'olive inférieure, des mécanismes de stabilisation sélective induisent le maintien d'une seule fibre grimpante par cellule. Cette réorganisation dépend de l'activité d'un autre système d'afférences, intrinsèque au cervelet, qui s'installe durant cette période entre les cellules de la couche des grains et les cellules de Purkinje (fibres parallèles). L'application d'APV dans le cervelet de rats nouveau-nés prévient cette réorganisation et les cellules de Purkinje restent donc, en l'absence de récepteurs NMDA fonctionnels, poly-innervées. Il semble donc que le récepteur NMDA participe ici directement au pro- 


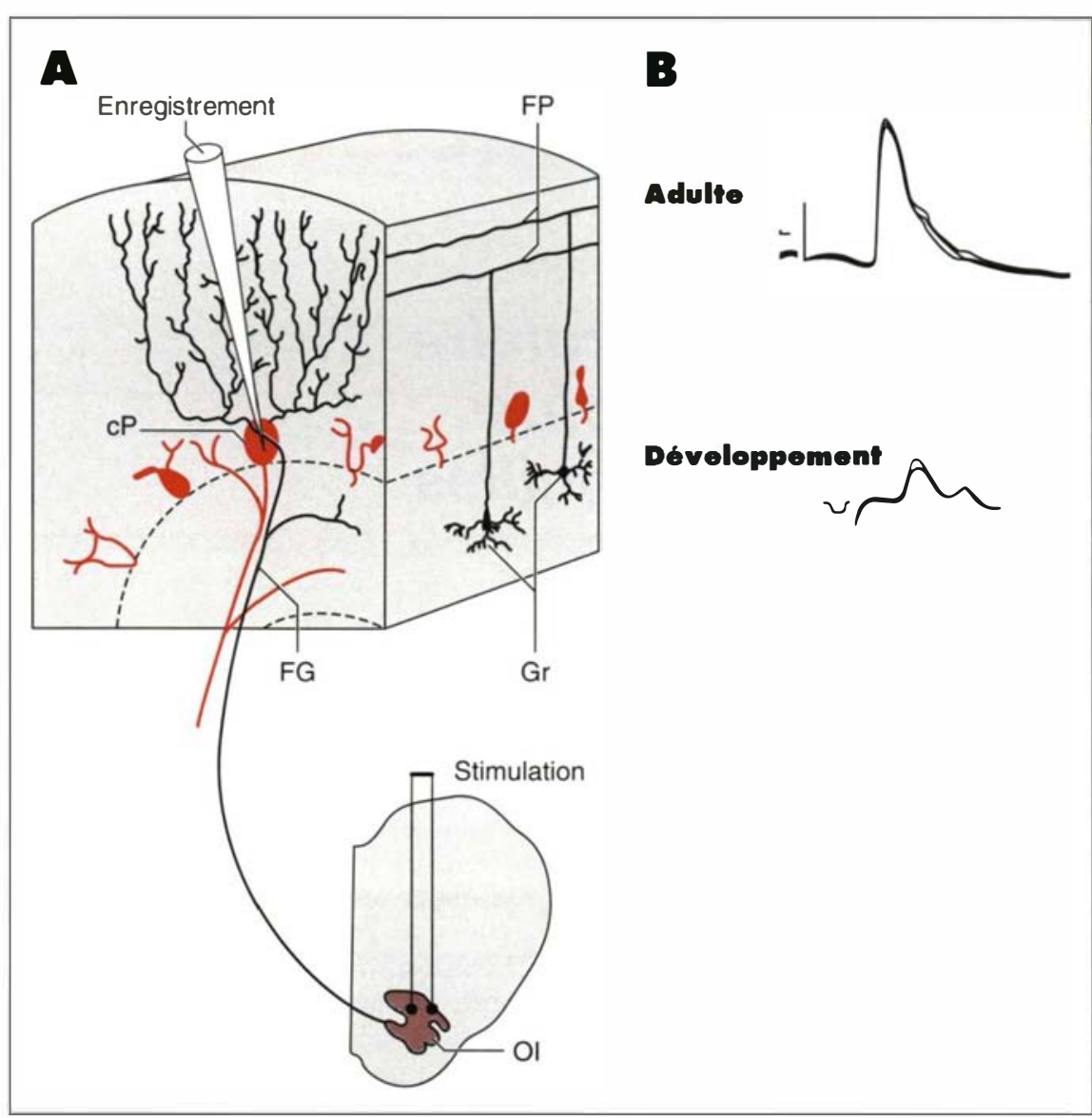

Figure 1. Organisation anatomo-fonctionnelle du cervelet. A. Schéma représentant deux des systèmes $d^{\prime}$ afférences aux cellules de Purkinje (cP) chez l'adulte: les fibres grimpantes (FG) qui proviennent de l'olive inférieure (OI) située dans le tronc cérébral, et les fibres parallèles (FP) qui naissent de la couche des grains (Gr). B. Effets de stimulations d'intensité croissante dans la voie des fibres grimpantes sur l'activité d'une cellule de Purkinje analysée par enregistrement intracellulaire. Au cours du développement, la poly-innervation se traduit par un recrutement progressif de fibres grimpantes qui apparaît sous la forme de potentiels successifs à l'enregistrement. Chez l'adulte, quelle que soit l'intensité (au-dessus du seuil), un seul potentiel est induit car une seule fibre grimpante contacte la cellule. cessus de stabilisation sélective des synapses et, par extension, à l'élimination de synapses fonctionnelles surnuméraires.

Les travaux sur le récepteur-NMDA convergent donc pour en faire une voie d'entrée de signaux nécessaires au remodelage des systèmes de connexion dans le système nerveux et, dans ce cadre, l'hypothèse anatomo-fonctionnelle émise il y a près de vingt ans par Changeux et Danchin trouve une base moléculaire solide. Il reste, toutefois, à identifier les mécanismes qui, à partir de cette voie d'entrée postsynaptique du signal, vont permettre un dialogue "constructif» (au plein sens du terme) entre les deux partenaires de la synapse.

M.P.

1. Changeux JP, Danchin A. Selective stabilization of developing synapses as a mechanism for the specification of neuronal networks. Nature 1976; 264: 705-11.

2. Constantine-Paton M, Cline HT, Debski E. Patterned activity, synaptic convergence and the NMDA receptor in the developing visual pathways. Annu Rev Neurosi 1990; 13 : 129-54 3. Lemarchandel V, Montagutelli X. La recombinaison homologue: de nouvelles pers pectives pour la transgénèse chez. les mammifères. médecine/sciences $1990 ; 6: 18-29$.

4. Li Y, Erzurumlu RS, Chen C, Jhaveri S, Tonegawa S. Whisker-related neuronal patterns fail to develop in the trigeminal brainstem nuclei of NMDARI knock-out mice. Cell 1994; 76 : 427-37.

5. Rabacchi S, Bailly Y, Delhaye-Bouchaud N, Mariani J. Involvement of the $\mathrm{N}$-methyl Daspartate (NMDA) receptor in synapse elimination during cerebellar development. Science 1992 ; 256: 1823-5. 